\title{
Electromagnetic excitations in nuclei: from photon scattering to photodisintegration
}

\author{
M. Erhard, C. Nair, R. Beyer, E. Grosse; A.R. Junghans , J. Klug, K. Kosev, \\ N. Nankov, G. Rusev, K.D. Schilling, R. Schwengner, and A. Wagner \\ Institut für Strahlenphysik \\ Forschungszentrum Dresden-Rossendorf \\ Postfach 510119 \\ 01314 Dresden \\ Germany \\ E-mail: A. Junghansefz-rossendorf.de
}

\begin{abstract}
In explosive nucleosynthesis temperatures are high enough for photodisintegration reactions to occur, e.g. leading to the production of p-process nuclei. In order to understand the reaction rates of element production and element disruption we started an experimental program at the new bremsstrahlung facility of the superconducting electron accelerator ELBE of FZ DresdenRossendorf. The bremsstrahlung facility and the detector setup are designed such that the scattering of photons from nuclei and the photodisintegration of nuclei around the particle separation energies can be studied under optimized background conditions. In activation measurements with bremsstrahlung at end-point energy from 10.0 to $16.5 \mathrm{MeV}(\gamma, \mathrm{p}),(\gamma, \mathrm{n})$ and $(\gamma, \alpha)$ reactions of ${ }^{92,100}$ Mo have been studied. Our activation yields can be described within a factor $2-3$ or better with calculations using the cross sections from recent Hauser-Feshbach models.
\end{abstract}

International Symposium on Nuclear Astrophysics - Nuclei in the Cosmos - IX

25-30 June 2006

CERN

\footnotetext{
*also at Institut für Kern- und Teilchenphysik, Technische Universität Dresden †peaker.
} 


\section{Introduction}

The 35 neutron deficient stable isotopes between $\mathrm{Se}$ and $\mathrm{Hg}$ that are shielded from the rapid neutron capture process by stable isobars, and bypassed by slow neutron captures of the s-process, are called p-process nuclei [1]. They are thought to be produced during supernova explosions through chains of photodisintegration reactions like $(\gamma, \mathrm{n}),(\gamma, \mathrm{p})$ and $(\gamma, \alpha)$ on heavy seed nuclei. The temperatures are in the region of $T=(1-3) \cdot 10^{9} \mathrm{~K}$. In many network calculations of the p-process nucleosynthesis, Mo and Ru isotopes are produced with lower abundances than observed in the solar system. This underproduction could indicate that a different nucleosynthesis process - the $v p$ process [2]- is responsible. Nucleosynthesis might be occuring in different astrophysical scenarios, e.g. He-accreting sub-Chandrasekhar-mass white dwarf explosions [3] or even gammaray bursts [4]. Production of Mo- and Ru p-process isotopes could also be enhanced by a higher ${ }^{22} \mathrm{Ne}(\alpha, \mathrm{n})^{25} \mathrm{Mg}$ cross section [5]. To test if the photodisintegration rates in the region of ${ }^{92} \mathrm{Mo}$ that are part of the nuclear physics input to the network calculations are correct, we have set up an activation experiment with bremsstrahlung from the new ELBE accelerator at Forschungszentrum Dresden-Rossendorf. We also did photon-scattering measurements with enriched Mo samples to study the dipole strength around the particle threshold, see ref. [6].

\section{Experimental Setup}

The new superconducting electron accelerator ELBE (for Electron Linear accelerator of high Brilliance and low Emittance) combines a high average beam current with a high duty cycle. It delivers electron beams of energies up to $40 \mathrm{MeV}$ with average currents up to $1 \mathrm{~mA}$ for experiments studying photon-induced reactions. The bremsstrahlung facility and the experimental area were designed such that the production of neutrons and the scattering of photons from surrounding materials is minimized [7]. A floorplan of the bremsstrahlung facility at ELBE is shown in Fig. 1. The primary electron beam is focussed onto a thin foil made from niobium with areal density adjustable between $1.7 \mathrm{mg} / \mathrm{cm}^{2}$ and $10 \mathrm{mg} / \mathrm{cm}^{2}$. After the radiator, the electron beam is separated from the photons by a dipole magnet and dumped into a graphite cylinder of $600 \mathrm{~mm}$ length and $200 \mathrm{~mm}$ diameter. A photoactivation site is located behind the beam dump where the available photon flux is up to $10^{10} \mathrm{~cm}^{-2} \mathrm{~s}^{-1} \mathrm{MeV}^{-1}$. For in-beam studies with bremsstrahlung, a photon beam is formed by a collimator made from high-purity aluminum placed inside the concrete shielding of the accelerator hall. Photons scattered from a target are observed by means of high-purity germanium (HPGe) detectors. The photon flux at the target position in the bremsstrahlung cave amounts up to $10^{8} \mathrm{~cm}^{-2} \mathrm{~s}^{-1} \mathrm{MeV}^{-1}$.

\section{Photoactivation measurements}

The number of radioactive nuclei $N_{a c t}\left(E_{0}\right)$ produced in a photoactivation measurement is proportional to the integral of the absolute photon flux $\Phi_{\gamma}\left(E, E_{0}\right)$ multiplied by the photodisintegration cross section $\sigma_{\gamma, x}(E)$ from the reaction threshold energy $E_{t h r}$ up to the bremsstrahlung spectrum end-point energy $E_{0}$.

$$
N_{a c t}\left(E_{0}\right)=N_{t a r} \cdot \int_{E_{t h r}}^{E_{0}} \sigma_{\gamma, x}(E) \cdot \Phi_{\gamma}\left(E, E_{0}\right) d E
$$




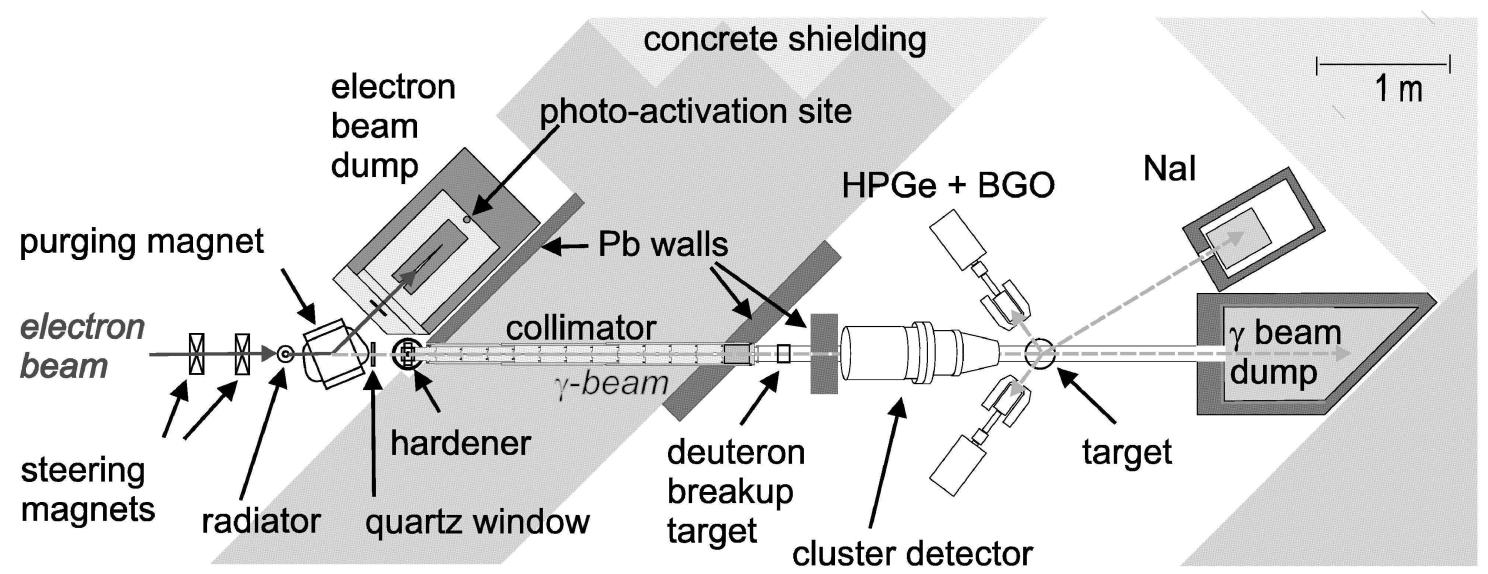

Figure 1: Bremsstrahlung facility and experimental area for photon-scattering and photodisintegration experiments at the ELBE accelerator. ${ }^{197} \mathrm{Au}$ and $\mathrm{H}_{3}{ }^{11} \mathrm{BO}_{3}$ or ${ }^{11} \mathrm{~B}$ samples were irradiated at the target position in the bremsstrahlung cave. ${ }^{n a t} \mathrm{Mo}$ and ${ }^{197} \mathrm{Au}$ samples were irradiated together at the photoactivation site.

The symbol $x=n, p, \alpha$ stands for the emitted particle. $N_{t a r}$ is the number of the target atoms in the sample. The number of radioactive nuclei $N_{a c t}\left(E_{0}\right)$ is determined experimentally after irradiation with bremsstrahlung in a low-level gamma-counting setup using an HPGe detector:

$$
N_{a c t}\left(E_{0}\right)=Y\left(E_{\gamma}\right) \cdot \kappa_{\text {corr }} /\left(\varepsilon\left(E_{\gamma}\right) \cdot p\left(E_{\gamma}\right)\right)
$$

$Y\left(E_{\gamma}\right), \varepsilon\left(E_{\gamma}\right), p\left(E_{\gamma}\right)$ are the dead-time and pile-up corrected full-energy peak counts of the observed transition, the absolute full-energy peak efficiency of the detector at the energy $E_{\gamma}$ and the emission probability of the photon with energy $E_{\gamma}$. The factor $\kappa_{\text {corr }}$ contains the relation of the detected decays in the measurement time $t_{\text {meas }}$ to the number of radioactive nuclei present. Decay losses in the time $t_{\text {loss }}$ in between the bremsstrahlung irradiation and the begin of the measurement as well as decay during the irradiation time $t_{i r r}$ are taken into account. The symbol $\tau$ denotes the mean life time of the radioactive nucleus produced during the photoactivation.

$$
\kappa_{\text {corr }}=\frac{\exp \left(t_{\text {loss }} / \tau\right)}{1-\exp \left(-t_{\text {meas }} / \tau\right)} \cdot \frac{t_{\text {irr }} / \tau}{1-\exp \left(-t_{\text {irr }} / \tau\right)}
$$

The constancy of the electron beam current and thus of the photoactivation rate was checked by monitoring both the electron current in the injector and in the beam dump. During a typical irradiation time of 8-16 hours there were no electron beam outages.

The sample (Mo) is activated in the high photon flux behind the beam dump together with a Au sample to measure an activation standard reaction, e.g. ${ }^{197} \mathrm{Au}(\gamma, \mathrm{n})$ (photoactivation site in Fig. 1). During the same experiment another Au sample is irradiated at the target position inside the bremsstrahlung cave. There, the absolute photon flux can be determined from the $\left(\gamma, \gamma^{\prime}\right)$ yield of a sample containing ${ }^{11} \mathrm{~B}$ which is irradiated in the same place. The cross section of ${ }^{197} \mathrm{Au}(\gamma, \mathrm{n})$ is then renormalized to give the measured activation yield with the absolute photon flux determined experimentally. With the renormalized ${ }^{197} \mathrm{Au}(\gamma, \mathrm{n})$ cross section and a simulated thick target bremsstrahlung spectrum, the absolute photon flux at the photoactivation site behind the beam dump can be determined. From the absolute photon flux and the measured activation yield the cross section normalization for photodisintegration of p-process nuclei like ${ }^{92}$ Mo can be determined. 


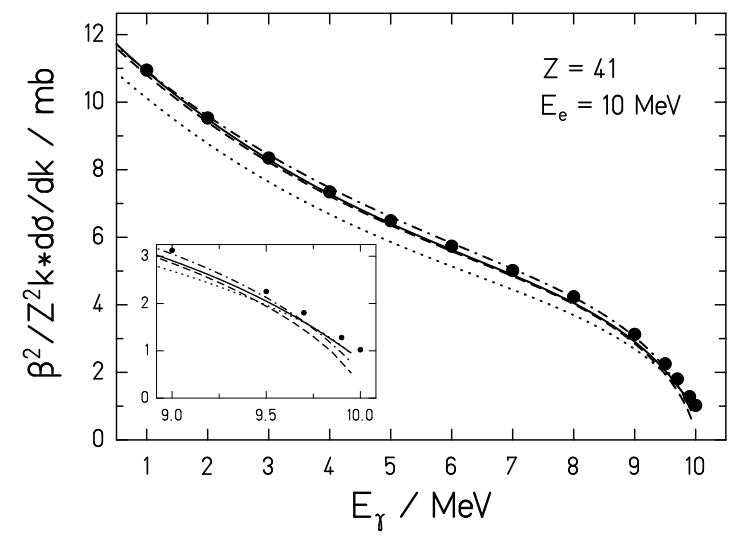

Figure 2: Theoretical bremstrahlung cross sections in comparison with the evaluation of Seltzer and Berger (circles)[8]. The full line is from ref. [11] the dashed line is from ref. [10]. They were calculated using a program from E. Haug [12] that also includes a screening correction according to ref. [13]. The dotted line is from the parametrization [14], the dash-dotted from Schiff [9].

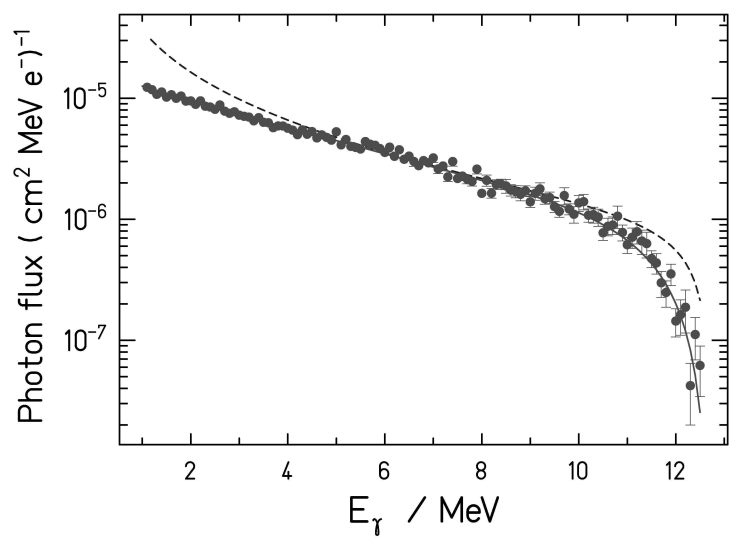

Figure 3: Bremsstrahlung spectrum at the main photoactivation site behind the graphite beam dump as calculated with MCNP 4C2 (circles) for an endpoint energy of $12.6 \mathrm{MeV}$. The dashed line denotes a theoretical thin target bremsstrahlung spectrum calculated according to Schiff [9]. It is normalized to the MCNP simulation at $6 \mathrm{MeV}$. The full line is a parametrization of the MCNP simulation.

\subsection{Photon spectrum and end-point energy determination}

The bremsstrahlung spectrum at the target position can be described well based on the bremsstrahlung cross section of a thin target. Fig. 2 shows theoretical bremsstrahlung cross sections compared to the evaluation of Seltzer and Berger [8] for a niobium radiator. The calculations were made using formulae given by Schiff [9], Heitler [10], and Roche [11]; the last two being corrected and programmed by Haug [12], who included an updated screening correction due to Salvat et al. [13]. Al Beteri et al. performed a theory motivated parametrization of experimental data as known in 1988 [14].

With the exception of ref. [14] the results agree to within $5 \%$ of each other at the low energy side of the spectrum, when atomic screening is taken into account. At the high energy side at about $\simeq 1 \mathrm{MeV}$ below the end point the descriptions differ to typically $20 \%$, see inset. This will influence the activation yield around the reaction threshold, where the yield integral depends strongly on the overlap with the high-energy tail of the photon spectrum. The data shown in this work were measured at $E_{0}>10.0 \mathrm{MeV}$ which is at least $2 \mathrm{MeV}$ above the respective ${ }^{197} \mathrm{Au}(\gamma, \mathrm{n})$ reaction threshold. The electron beam energy has been determined using a dipole magnet in the beamline with a well known field integral measuring the beam positions before and after the magnet. With MCNP4C2[15] we have calculated the absolute photon flux at the photoactivation site behind the beam dump, this analysis is in progress. Based on a parametrization of the MCNP results the thicktarget photon spectral shape was calculated and used in the analysis of our Mo photoactivation data, see Fig. 3.

\section{2 ${ }^{197} \mathrm{Au}(\gamma, \mathbf{n})$ as activation standard}

${ }^{197} \mathrm{Au}$ samples of mass $65 \mathrm{mg}$ and $200 \mathrm{mg}$ have been irradiated at end-point energies of 


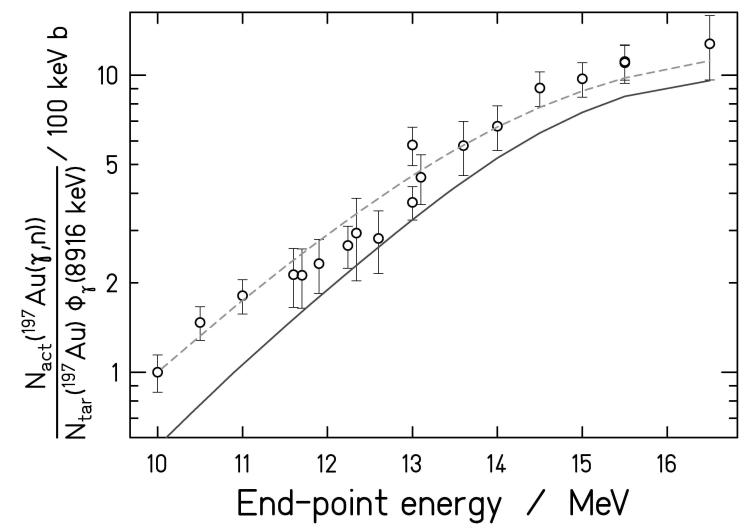

Figure 4: Preliminary activation yield of ${ }^{197} \mathrm{Au}(\gamma, \mathrm{n})$ measured at the target position, see Fig. 1. The experimental yield is normalized to the number of ${ }^{197} \mathrm{Au}$ atoms and to the absolute photon flux at the energy $E_{\gamma}=8916 \mathrm{keV}$. The data are compared to yield integrals computed with the cross sections from TALYS and NON-SMOKER using the absolute photon flux determined from known transitions in a sample containing ${ }^{11} \mathrm{~B}$.

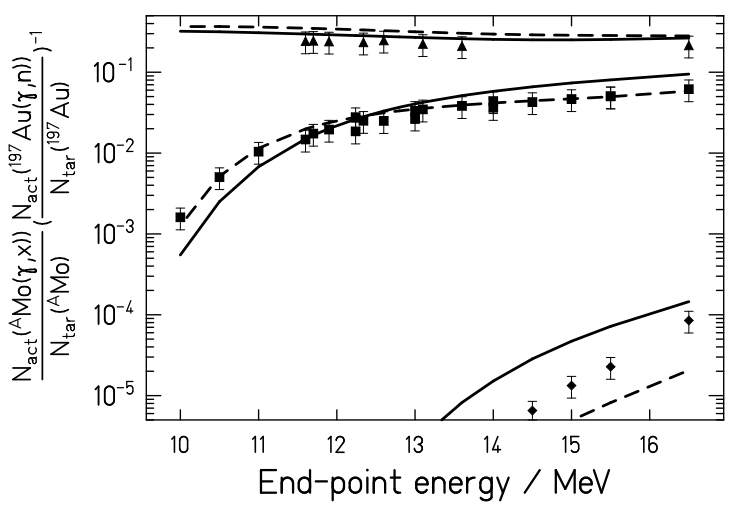

Figure 5: Measured activation yields at the photoactivation site for different Mo-Isotopes normalized to the activation yield from ${ }^{197} \mathrm{Au}(\gamma, \mathrm{n})$ irradiated simultaneously and to the number of the respective target atoms as a function of the bremsstrahlung endpoint energy. The full symbols denote experimental yields from ${ }^{100} \operatorname{Mo}(\gamma, \mathrm{n})$ (triangles), ${ }^{92} \operatorname{Mo}(\gamma, \mathrm{p})+$ $(\gamma, n)$ (squares), and ${ }^{92} \operatorname{Mo}(\gamma, \alpha)$ (diamond). The lines are calculated with photodisintegration cross sections from ref. [18] (full) and ref. [19] (dashed).

10.0 $\mathrm{MeV}$ up to $16.5 \mathrm{MeV}$ at the target position together with an $\mathrm{H}_{3}{ }^{11} \mathrm{BO}_{3}$ sample, mass 2.93 $\mathrm{g}$, enriched in ${ }^{11} \mathrm{~B}$ to $99.27 \%$ or with a $99.52 \%$ enriched sample of ${ }^{11} \mathrm{~B}$ of mass $4.51 \mathrm{~g}$. The absolute photon flux was determined using known transition strengths[16] in ${ }^{11} \mathrm{~B}$ at $E_{\gamma}\left(\Theta_{l a b}=90^{\circ}\right)=$ 4444, 5019, 7283 and $8916 \mathrm{keV}$, respectively. Detectors were positioned at $\Theta_{l a b}=90^{\circ}$ and $\Theta_{l a b}=$ $127^{\circ}$. Angular correlation effects are important for the ${ }^{11} \mathrm{~B}$ photon scattering yields as observed especially at $90^{\circ}$. Feeding corrections are estimated to be small for the high-energetic transitions (no feeding for the highest transition) used here, but will be included in the final data analysis.

The number of ${ }^{196} \mathrm{Au}$ nuclei produced during the activation was determined from decay measurements in a low-level counting set up with HPGe detectors. The absolute ful-energy peak efficiency was measured with the help of several calibration sources from Amersham and PTB [17] to $3 \%$ uncertainty in the energy range from $150-2000 \mathrm{keV}$. A Cd absorber was used to minimize coincidence-summing effects with $\mathrm{x}$-rays emitted from the Au samples and some of the calibration sources. The efficiency was checked by GEANT3 simulations that were adjusted to the experimental data to give the efficiency as a function of photon energy. Coincidence-summing corrections for the $333 \mathrm{keV}$ and $356 \mathrm{keV}$ decay lines of ${ }^{196} \mathrm{Au}$ were taken into account. The weaker transition at $426 \mathrm{keV}$ into $\mathrm{Hg}$ that does not have coincidence summing was included in the analysis. The number of nuclei produced during the activation at the target position was normalized to the number of ${ }^{197} \mathrm{Au}$ target nuclei and to the photon flux at $E_{\gamma}=8916 \mathrm{keV}$. The data for ${ }^{197} \mathrm{Au}(\gamma, \mathrm{n})$ are shown in Fig. 4 in comparison with results that were calculated using the absolute photon flux determined experimentally and the theoretical ${ }^{197} \mathrm{Au}(\gamma, \mathrm{n})$ cross section from the TALYS program [19] and from NON-SMOKER [18]. The theoretical result from TALYS agrees well with the experimental data. The combined effect of the systematic uncertainties in the absolute photon flux related to the 
end-point energy, spectral shape of bremsstrahlung and electron-beam energy resolution, have not been finally determined yet. About $20 \%$ uncertainty does seem to be realistic, however. From the data shown in Fig. 4 we conclude that the results from the NON-SMOKER code are considerably lower than observed experimentally. The yield integrals calculated with NON-SMOKER are only 60 to $80 \%$ of the yield integrals calculated with TALYS.

\subsection{Photoactivation measurements of Mo-Isotopes}

Natural samples of molybdenum (mass 0.66 - $4.80 \mathrm{~g}$, disc diameter $20 \mathrm{~mm}$ ) have been irradiated together with the Au samples as discussed above. As the absolute normalization of the photon flux at the photoactivation site is still in progress, Fig. 5 shows the measured reaction yields relative to the experimental Au reaction yield as calculated in eq. (3.2). The data are normalized to the different number of target atoms in the samples. The experimental data points are compared with the yield integrals calculated with a simulated thick-target bremsstrahlung spectrum and the NON-SMOKER and TALYS photodisintegration cross sections. The yield integrals (eq. 3.1) are calculated relative to the ${ }^{197} \mathrm{Au}(\gamma, \mathrm{n})$ yield integral.

The data agree on a scale relative to ${ }^{197} \mathrm{Au}(\gamma, \mathrm{n})$ to typically $20-30 \%$ with the simulation. The ${ }^{92} \mathrm{Mo}(\gamma, \mathrm{p})$ reaction cross section is dominant at energies below $12.6 \mathrm{MeV}$, as the ${ }^{92} \mathrm{Mo}(\gamma, \mathrm{n})$ channel is not open yet. The ${ }^{91 m} \mathrm{Nb}$ nuclei produced were identified by the $1205 \mathrm{keV}$ transition following the EC decay into ${ }^{91} \mathrm{Zr}$. The population of the long lived $\left(t_{1 / 2}=680 \mathrm{yr}\right)$ ground state in ${ }^{91} \mathrm{Nb}$ that cannot be easily detected in an activation measurement has to be taken into account, e.g. by statistical model calculations. With the TALYS code we have calculated that this effect is around 5 - $10 \%$ in the energy range measured. The contribution from ${ }^{92} \mathrm{Mo}(\gamma, \mathrm{n})$ above $12.6 \mathrm{MeV}$ produces shorter lived activity that can be identified with a pneumatic delivery system recently installed at ELBE. The ${ }^{100} \operatorname{Mo}(\gamma, n)$ results will be used for comparison with Coulomb dissociation experiments done at GSI, Darmstadt [20]. We observed the ${ }^{92} \operatorname{Mo}(\gamma, \alpha)$ reaction with samples of natural Mo at the rather low end-point energy of $14.5 \mathrm{MeV}$. In addition, also ${ }^{144} \mathrm{Sm}(\gamma, \alpha)$ has been observed at end point energies of $15.1 \mathrm{MeV}$ and $16.0 \mathrm{MeV}$ using natural $\mathrm{Sm}_{2} \mathrm{O}_{3}$ samples. The similarity of the relative data shown in Fig. 5 as compared to the NON-SMOKER data [18] and TALYS data [19] suggest already that the predicted underproduction of $\mathrm{Mo} / \mathrm{Ru}$ isotopes might not be due to wrong photodisintegration rates. Absolute data currently under analysis will allow to draw a firm conclusion.

\section{Conclusion}

First photodisintegration measurements of the p-process nucleus ${ }^{92}$ Mo have been performed at the new bremsstrahlung facility at ELBE, FZ Dresden-Rossendorf. The activation technique has been used to identify the different reaction products by $\gamma$-decay spectroscopy. The photodisintegration reactions $(\gamma, \mathrm{n}),(\gamma, \mathrm{p})$, and $(\gamma, \alpha)$ have been observed. The absolute photon flux has been measured online by known transitions in ${ }^{11} \mathrm{~B}$ and by using the reaction ${ }^{197} \mathrm{Au}(\gamma, \mathrm{n})$ as an activation standard. Preliminary results indicate that the absolute cross section of ${ }^{197} \mathrm{Au}(\gamma, \mathrm{n})$ as calculated by the NON-SMOKER code is lower than the experimental data. This was also found in the energy region below $10 \mathrm{MeV}$ in ref. [21]. The reaction yields for ${ }^{92} \mathrm{Mo}(\gamma, \mathrm{p})+(\gamma, \mathrm{n})$ relative to ${ }^{197} \mathrm{Au}(\gamma, \mathrm{n})$ 
agree to within $20-30 \%$ with the model calculations [18]. These reactions contribute to the possible destruction of the p-process nucleus ${ }^{92} \mathrm{Mo}$.

\section{Acknowledgments}

We thank P. Michel and the ELBE-crew for providing stable, high-intensity electron beams and A. Hartmann and W. Schulze for continuous valuable technical support. We gratefully acknowledge theory discussions and help by H.W. Barz and E. Haug.

\section{References}

[1] M. Arnould, S. Goriely, Phys. Rep. 384, 1 (2003).

[2] C. Fröhlich et al., Phys. Rev. Lett. 96142502 (2006).

[3] S. Goriely et al., A\&A 444 L1 (2005).

[4] R. Surman, G. C. McLaughlin, and W. R. Hix, ApJ 6431057 (2006).

[5] V. Costa et al., A\&A 358, L67 (2000).

[6] G. Rusev, E. Grosse et al., Eur. Phys. J A 27, s01,171 (2006).

[7] R. Schwengner et al., NIM A 555211 (2005).

[8] S. M. Seltzer and M. J. Berger, Atom. Data and Nuc. Data Tables 35, 345 (1986).

[9] L.I. Schiff, Phys. Rev. 83, 252 (1951).

[10] W. Heitler, The quantum theory of radiation, (Dover publications, New York 1984) p. 242.

[11] G. Roche, C. Ducos, J. Proriol, Phys. Rev. A 5, 2403 (1972).

[12] E. Haug, private communication.

[13] F. Salvat, J. D. Martinez, R. Mayol, J. Parellada, Phys. Rev. A 36, 467 (1987).

[14] A. A. Al-Beteri, D. E. Raeside, Nucl. Instr. Meth. B 44, 149 (1989).

[15] MCNP4C2: http://www.nea.fr/abs/html/ccc-0701.html

[16] F. Ajzenberg-Selove, Nucl. Phys. A 506, 1 (1990).

[17] Physikalisch Technische Bundesanstalt, Fachbereich 6.1, Bundesallee 100, Braunschweig, Germany; Amersham: ISOTRAK AEA Technology QSA, Gieselweg 1, Braunschweig, Germany.

[18] T. Rauscher, F.-K. Thielemann, Atomic Data and Nuclear Data Tables 88, 1 (2004).

[19] A. J. Koning, S. Hilaire, M. C. Duijvestin, Proc. Int. Conf. Nucl. Data for Science and Technology, ND2004, Santa Fe, USA, 2004, AIP Conference proceedings 769, 177 (2005).

[20] S. Müller et al., PoS(NIC-IX) 57 (2006).

[21] K. Vogt et al., Nucl. Phys. A 707, 241 (2002). 